\title{
Corela
}

Cognition, représentation, langage

HS-19 | 2016

Le point de vue pris au mot

\section{Variabilité versus stabilité relative du rapport forme / sens dans nos langues : entre synchronie et diachronie}

\section{Serguei Sakhno}

\section{(2) OpenEdition \\ Journals}

Édition électronique

URL : http://journals.openedition.org/corela/4374

DOI : 10.4000/corela.4374

ISSN : 1638-573X

Éditeur

Cercle linguistique du Centre et de l'Ouest - CerLICO

Référence électronique

Serguei Sakhno, «Variabilité versus stabilité relative du rapport forme / sens dans nos langues : entre synchronie et diachronie », Corela [En ligne], HS-19 | 2016, mis en ligne le 08 juin 2016, consulté le 19 avril 2019. URL : http://journals.openedition.org/corela/4374; DOI : 10.4000/corela.4374

Ce document a été généré automatiquement le 19 avril 2019

\section{(c) (i) (2)(2)}

Corela - cognition, représentation, langage est mis à disposition selon les termes de la licence Creative Commons Attribution - Pas d'Utilisation Commerciale - Partage dans les Mêmes Conditions 4.0 International. 


\title{
Variabilité versus stabilité relative du rapport forme / sens dans nos langues: entre synchronie et diachronie
}

\author{
Serguei Sakhno
}

\section{Introduction}

1 Dans les langues, à côté des nombreuses irrégularités lexico-sémantiques et de l'opacité fréquente du signifiant (rapport forme / sens), il y a des régularités observées en diachronie et en synchronie : régularités dans les façons dont telle(s) forme(s) exprime (nt) tel(s) sens et inversement, tel(s) sens est (sont) rendu(s) par telle(s) forme(s), ainsi que des analogies constatées dans l'évolution sémantique des mots aboutissant à des modèles similaires de polysémie.

2 Par ailleurs, un sémanticien doit tenir compte des représentations sociales prédominantes: face à ces représentations stéréotypées, les mots «cristallisent des points de vue", selon l'heureuse formulation de Raccah (2011). En effet, les mots ne décrivent pas le monde mais des rapports au monde (Nemo 2002): les lexèmes ne décrivent les objets que dans la mesure où ceux-ci s'inscrivent dans des relations. L'objectivité de la «mise en mot» du monde n'est jamais acquise : appeler un ministère ministère de la guerre ou ministère de la défense est aussi sémantiquement marqué que le fait d'appeler la capitale d'un pays capitale (fr.), Hauptstadt (allemand : « chef-ville ») ou stolica (russe : « ville du trône »).

3 Les faits à décrire et à systématiser sont de trois types :

- régularités à caractère morphologique dérivationnel : à titre d'exemple, on peut s'intéresser aux suffixes diminutifs à sens méronymique ("partie de $\mathrm{N} »)$ : fr. manche (fém.) > manch-ette (< « partie d'une manche »); russe lampa 'lampe' > lampočk- $a$ 'ampoule' (< «partie essentielle d'une lampe » < « petite lampe »), exemple de Mel'čuk (1993: 307-308) ${ }^{1}$;

- régularités liées à l'évolution du sens et à la polysémie (parallèles de dérivation 
sémantique : 'saisir' > 'comprendre', cf. fr. com-prendre, italien capire 'comprendre' ; 'respirer' > 'âme, principe vital', cf. fr. spir-ituel et re-spir-er) ;

- régularités de type mixte, relevant à la fois de la dérivation morphologique et de la dérivation sémantique : ce cas, sans doute le plus fréquent, nous intéresse en particulier.

Ces questions restent peu étudiées dans une optique typologique et elles sont insuffisamment formalisées. L'analyse du lexique de telle ou telle langue et la comparaison des langues (indépendamment de leur parenté ou au contraire, en rapport avec leur parenté, ainsi que compte tenu des contacts linguistiques qui aboutissent souvent à des calques) fournissent une profusion de données à complexité effrayante : on est souvent entre régularités et irrégularités, dans une variabilité redoutable du rapport forme / sens. Bref, un abîme, au sens étymologique (gr. a-byssos 'sans fond') ! Et les limites des rationalisations lexico-sémantiques à base d'étymologie ne sont pas bien définies.

Malgré la démotivation généralement postulée pour le lexique (postulat en partie discutable, qui doit être nuancé), on observe souvent une transparence relative du signifiant, mais qui se manifeste à divers degrés et dont l'analyse est souvent problématique. Même dans des cas qui paraissent simples, la construction du sens ne va pas de soi : déterrer $=$ dé- + terre( $r$ ) suppose un schéma distinct ('retirer qqch. de terre') de celui de déneiger, dépoussiérer ('enlever la neige / la poussière de la surface de qqch.'), comme le montre Nemo (2011).

Pour Jalenques (2000), dans fr. regarder, il y a re- et -gard(er) : chacun de ces morphèmes conserverait, d'un certain point de vue, son identité sémantique : re-garder $=r e^{-}+$garder 'surveiller, avoir l'oeil sur' + 'retour à un état d'être en contact visuel'. Selon Franckel (1994), le schéma sémantique est le suivant: «Re-P renvoie à une double construction d'une occurrence, la $1^{\text {re }}$ construction de $\mathrm{P}$ étant purement temporelle, alors que la $2^{\mathrm{e}}$ construction se trouve spécifiée par la $1^{\text {re }}$, ce qui permet de mettre en place des formes de stabilisation quantitative $»^{2}$. Ce qui revient à relever le rôle « intensif » et « idéalisant » du préverbe re- en français, cf. marquer - remarquer, sembler - ressembler, tenir - retenir, unir réunir, etc. Le rôle de ce préfixe est à prendre en compte, en synchronie et en diachronie, dans l'explication de la variation sémantique des lexèmes de nos langues, liés à la perception visuelle: cf. it. guardare 'regarder', 'prendre soin de', 'faire attention' et 'garder, préserver', fr. garder et re-garder, angl. regard 'respect', fr. ré-vér-er, ré-serv-er, all. warten 'garder ; attendre' et angl. re-ward 'récompense' (< racine i.-eu. *swer-, variantes *ser- et *wer- 'surveiller, épier, observer') ; fr. re-spect-er (< i.eu. *spek- 'faire attention, observer').

7 De nombreuses régularités se présentent comme des faits de submorphologie (Bottineau $2008,2010,2012)$ ou de polymorphie : selon Nemo (2003), un locuteur français moyen va rapprocher couler - découler et dégouliner; rotation et tourner, en dépit de l'absence de lien étymologique « savant ».

8 Notre approche se situe dans le prolongement des analyses en sémantique synchronique et diachronique des linguistes cités, ainsi que dans le prolongement de certains de nos propres travaux (Sakhno 2001 ; Sakhno et Hénault-Sakhno 2001, 2005).

\section{Formes analysables ou non? Comparables ou non?}

9 À l'époque de l'accroissement des échanges et des communications à l'échelle européenne et planétaire, la question du plurilinguisme doit se poser en termes de «transparence » 
des langues entre elles par opposition à leur « opacité » relative. La transparence entre les principales langues européennes dites «occidentales» est assez importante: elle est assurée par leur «matière expressive» (lexique, modèles syntaxiques, discursifs, rhétoriques), - autrement dit leur corpus - matière qui est unifiée et standardisée en profondeur. Ainsi, un francophone n'est jamais complètement dépaysé au contact de l'italien, langue romane assez proche du français. On peut penser aussi aux nombreuses similitudes existant entre le français et l'anglais, seraient-elles dues au double héritage linguistique gréco-latin - sans oublier l'héritage culturel judéo-chrétien - ou aux contacts intenses au cours des siècles (aboutissant notamment à de nombreux emprunts directs ou indirects). Il est bien connu notamment que l'anglais, langue germanique, a emprunté la moitié de son vocabulaire au français, langue romane. Ces ressemblances et analogies, voire identités, facilitent l'apprentissage des deux langues et la communication entre les francophones et les anglophones.

Mais la transparence n'est jamais absolue (pensons aux mots anglais ne ressemblant en rien à leurs équivalents français, ainsi qu'aux nombreux " faux amis »), et elle est même assez problématique pour certaines langues (polonais, lituanien, finnois) du point de vue des francophones. Même une langue très proche du français comme l'italien comporte des énigmes. Quand on séjourne en Italie, on voit partout des enseignes affichant NOLEGGIO. On finit par apprendre que cela signifie 'location (de voitures)'. Mais la forme du mot parait surprenante : pourquoi location se dit en italien noleggio?

On pourrait penser, en suivant le principe de l'« étymologie populaire », que dans le mot italien noleggio 'location d'un moyen de transport', no- est une négation et que -leggio est à rattacher au mot italien legge 'loi'. L'imagination aidant, on arrive à la conclusion: les loueurs de voitures en Italie sont malhonnêtes, ils agissent en dehors de la loi. Il y a de quoi devenir paranoïaque! Malheureusement (ou contraire, heureusement), ce raisonnement un peu naif est complètement faux: le mot en question a une origine différente.

En réalité, ce mot italien, qui parait si étrange, correspond exactement, du point de vue de sa racine, au verbe français noliser qui signifie 'affréter, louer' (en parlant d'un bateau). Ce terme de marine est relativement peu connu. Noleggio et noliser remontent tous deux au mot latin naulum 'fret' qui est lui-même d'origine grecque, du grec naulon 'prix du transport en bateau; fret'. Plusieurs mots français font partie de la même famille étymologique : naval, naviguer, nef, naufrage, nausée. Il existe un autre emploi technique de noliser : un avion nolisé est ce qu'on appelle couramment un charter.

Par ailleurs, l'étymologie gréco-latine de noleggio / noliser est révélatrice d'un trait historique important de la civilisation du monde méditerranéen : les Romains, qui étaient au début de mauvais navigateurs, ont appris des Grecs l'art de la navigation en assimilant des termes grecs correspondants. L'utilité du rapprochement entre it. noleggio et fr. noliser est certaine : on comprend mieux le mot italien et on mémorise mieux le mot français qui est relativement rare et technique.

Même à l'intérieur d'une langue, la transparence des rapports entre les formes et leurs sens est souvent relative. N'importe qui peut mettre en rapport banc 'siège' et banquette: même si ce dernier mot (issu de l'ancien provençal banqueta 'petit banc', 'siège à plusieurs places') ne peut pas désigner par exemple un petit banc de jardin public, il est tout naturellement perçu par un Français comme renvoyant à une sorte de banc. Le rapprochement avec l'adjectif bancal est plus difficile, mais non impossible (d'après la 
divergence des pieds d'un banc, on pense à une personne boiteuse ou à un objet mal équilibré : meuble bancal). Quant au rapport entre banc et banque ou banquet, il est encore moins certain pour un francophone. Mais ce rapport peut être réactivé, réactualisé au contact d'autres langues :

- sur le plan à la fois de la forme et du sens (italien banco, espagnol banca, banco, allemand Bank 'banc, banquette' et 'banque');

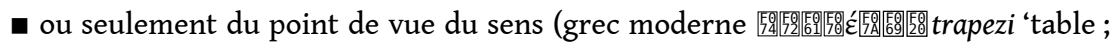

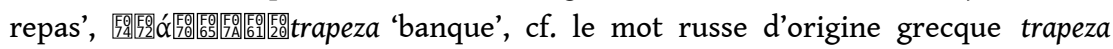
'repas').

15 À ce propos, on doit rappeler que le mot banc garde une trace de son ancien rapport avec le domaine du commerce et de la finance, puisque certaines variantes du français régional connaissent l'emploi de banc au sens de 'étal d'un marchand'. De ce point de vue, les données des autres langues européennes nous aident à mieux comprendre les particularités du mot français en question.

L'étymologie dite "naïve» ou "populaire» (termes injustement ressentis comme péjoratifs) joue un rôle très important l'activité langagière. Ainsi, le mot français banquise, dont l'origine est distincte de celle de banc (car il vient du scandinave, cf. suédois packis, danois pakis ou norvégien pakkis 'amas de glace'), a été rapproché par les locuteurs francophones de banc au sens de 'amas (de sable, de neige, de poissons)', mot historiquement lié à banc 'siège'. La "forme interne" de banquise est donc ressentie comme se trouvant en rapport avec banc dans tous ses sens. L'anglais peut conforter un locuteur francophone dans cette possibilité de rapprochement (cf. bank 'talus ; bord, rive, berge ; amoncellement ; banc de sable', snow bank 'congère' et bank 'banque').

17 La variabilité du rapport forme / sens dans nos langues coexiste avec une stabilité relative de ce même rapport. Certaines configurations sémio-formelles posent un vrai problème de cohérence dans la perspective d'une description sémantique et morphologique située entre synchronie et diachronie.

En synchronie, fr. contrée, angl. country ne sont pas immédiatement associables à fr. contre (-), angl. counter-. Cependant, ils remontent à lat. contrata regio 'région opposée, région d'en face' < contra 'contre, en face'. À titre de parallèle sémantique, cf. all. Gegend 'région, contrée' < gegen 'contre, en face', gallois ardal 'pays; frontière, marche' < ar 'sur' + tal 'front, ce qui est en face; fin'. Sémantiquement, le rapprochement de ce lien sémioformel est possible avec le rapport " frontière > région frontalière > contrée, pays ", cf. lat. fines 'pays' < finis 'fin, bout' ; v. irl. mruig 'région', breton bro 'région, contrée, pays' < celt. *mrog- < i.eu. *morg- 'limite', cf. lat. margo (> fr. marge), germ. mark- (> fr. marche 'frontière', marquis, Dane-mark, etc.). On notera également le toponyme Ukraine 'bord, périphérie' < russe / ukrainien Ukraina / Ukrajna < russe kraj 'bord, périphérie', cf. o-kraina 'périphérie (d'une ville)'; russe strana 'pays' lié à storona '(autre) côté', lituanien kraštas 'région' < 'côté, bord'.

19 En revanche, dans fr. rencontrer ( = re-en-contr-er), l'élément contre(-) est bien plus visible, cf. fr. à l'encontre de (< lat. tard. in-contra), ainsi que all. begegnen 'rencontrer' ( = be-gegn-en ), néerl. tegenkomen 'rencontrer' ( = tegen-komen).

20 Mais fr. contrôler constitue un cas assez complexe : étymologiquement, quoique d'une façon non transparente pour un locuteur moyen, ce verbe est lié d'une part à fr. enrôler ; enrouler; rôle; rouler, etc. D'autre part, contrôler sera rapproché en diachronie (et éventuellement en synchronie, pour beaucoup de locuteurs) de contredire. Mais un linguiste diachronicien ne va pas le rapprocher de contraindre, contracter, contribuer, 
malgré l'illusion formelle en synchronie, car ces derniers verbes s'analysent en diachronie comme con-traindre, con-tracter, con-tribuer < lat. con-stringere, con-trahere, contribuere.

En conséquence, historiquement, contrôler se présente comme contre-roller, du latin médiéval contra-rotulare 'vérifier un compte sur un registre tenu en double (permettant la vérification)' < lat. contra-rollus / contra-rotulus 'registre tenu en double'. Cette formation est (seulement en partie) analogue à all. gegenüberstellen ( = gegen-über-stellen) 'comparer, rapprocher, mettre en face, opposer, mettre en regard', mais l'allemand n'a pas de * gegenrollen! (cf. en revanche Rollmops 'hareng roulé').

Quant à l'élément contre- dans contre-roller, il convient de rappeler ici son origine : lat. contra 'en face de, vis-à-vis de, dans la direction de, etc.' < con-tr- $a$, avec un suffixe -trmarquant l'opposition de deux notions (cf. extra $=$ ex-tr- $a$, alter $=$ al-ter, en regard de alius).

Du point de vue du sens de 'mettre en face, opposer, mettre en regard', contre-roller peut être rapproché de fr. ci-contre 'en regard' ainsi que de l'un des emplois de la préposition contre 'vers' (français de Suisse : aller contre la ville).

Le second élément de contre-roller est issu de lat. médiéval rollus 'rouleau (parchemin roulé)' < rotulus, diminutif de lat. rota 'roue' (> fr. roue, rotation), d'où par ailleurs fr. rôle 'parchemin contenant qqch. d'écrit' > 'liste, énumération détaillée' (aujourd'hui vocabulaire juridique et administratif), 'partie d'un texte que doit dire un acteur', cf. également fr. enrôler 'inscrire sur un rôle', 'engager dans une troupe, un parti, etc. (en inscrivant le nom sur un document)'. Notons que fr. rouler est dans la même famille étymologique ( $<$ ancien fr. roelle 'petite roue', cf. fr. rouelle < lat. rotula, autre diminutif de rota, cf. fr. rotule), rota < i.-eu. *roth- 'roue', cf. all. Rad 'roue'.

À titre de parallèle sémantique, pour justifier le sens du lat. médiéval rollus 'rouleau (parchemin roulé)', on doit citer fr. volume < lat. volu-men 'chose enroulée; rouleau d'un manuscrit' < lat. volvere 'tourner'. Pour le sens 'vérifier' de contrôler, on doit tenir compte du paradigme constitué par les composés français avec contre- tels que contre-expertise, contre-enquête, contre-interrogatoire, contre-essai, contrepartie ('double d'un registre' > 'écritures qui servent de vérification'), contre-épreuve ('double comptage'), contreassurance, contre-seing ; contre-réaction 'action de contrôle en retour, feedback', contre-digue 'ouvrage destiné à consolider la digue principale', contre-allée, contre-amiral.

C'est dans cette double optique qui dépasse une séparation trop absolue entre synchronie et diachronie que peut être décrite la configuration sémio-formelle complexe de fr. contrôler.

\section{Quelles sont les limites des rationalisations lexico- sémantiques à base d'étymologie?}

Selon Rey-Debove (1998: 199), l'étymologie de fr. route, du lat. via rupta 'voie frayée, rompue', n'aurait aucune pertinence linguistique pour un francophone d'aujourd'hui : il ne s'agirait plus que d'une anecdote historique, les routes étant destinées à relier les villes. Pourtant, la parenté entre route et rompre, rupture, du latin rumpere, n'est pas inintéressante du point de vue du français moderne. En effet, l'étymologie de fr. route (< latin via rupta 'voie ouverte, pratiquée' ou 'voie recouverte de pierres cassées') permet de comprendre son sémantisme en synchronie ainsi que la construction de son sens; notamment, cela permet d'expliquer le fait que ce mot implique 'voie bien tracée, 
aménagée', 'voie praticable', par rapport à son synonyme relatif chemin (d'origine celtique), cf. route nationale, autoroute mais chemin rural, chemin de halage, ${ }^{*}$ autochemin.

Par ailleurs, on peut tenir compte des dérivés de route en diachronie, cf. fr. routine 'chemin très fréquenté' > 'savoir-faire acquis par une pratique prolongée' > 'action machinale'.

On soulignera l'importance des parallèles sémantiques en diachronie avec des lexèmes liés au sens 'voie bien tracée, aménagée' dans d'autres langues :

- ital. strada, angl. street, all. Strasse 'route; rue' < lat. via strata 'voie pavée' < sternere 'étendre, étaler, etc., cf. sternum 'os plat', fr. strate, le stratifié (revêtement de sol), etc.;

- russe doroga 'route' < 'passage pratiqué dans la forêt en arrachant les arbres et d'autres végétaux', cf. russe drat' 'arracher' < i.-eu. *der- 'arracher', lié étymologiquement à grec derma 'peau' (< derein 'écorcher, dépouiller' : mauvaise référence pour une publicité de produits cosmétiques!);

- tchèque cesta 'route, voie ; voyage' < slave *kes- 'lisser, raser, gratter' et 'peigner', cf. russe kosit' 'faucher (l'herbe)', česat' 'gratter', i.-eu. *kes- / *kos- / *kas- 'couper', cf. lat. castrare 'couper, émonder', angl. hards et all. Hede 'étoupe';

- v. scand. braut 'route, voie' < brjöta 'rompre, briser, casser'.

Voici un autre exemple, plus complexe: Paulhan (1988) affirme l'inutilité du rapport diachronique fr. salaire < lat. salarium < sal, salis 'sel' ('ration de sel, somme donnée aux soldats pour acheter du sel') : ce lien seraint sans intérêt pour les locuteurs d'aujourd'hui.

Bien au contraire, le lien est sémantiquement pertinent, car cela nous renvoie à une époque ancienne où le sel était rare et apprécié, et les références culturelles sont importantes ${ }^{3}$. Il suffit de penser aux toponymes tels que Salzbourg, Hallstadt (Autriche), Halle (Allemagne), aux richesses issues du commerce du sel (exemple de Guérande, en Loire-Atlantique), aux citations bibliques (cf. Vous êtes le sel de la terre... - analysé par Wierzbicka (2000)).

Mais les points de vue au sens de Raccah (2011) associables à sel sont variables. Les faits sémantiques en synchronie et en diachronie sont les suivants :

- fr. sel, emploi fig. 'ce qui donne du piquant, de l'intérêt' (sel attique, plaisanterie pleine de sel); mais sel peut donner lieu à des points de vue négatifs, cf. fr. saler 'punir qqn'(argot, 1930), addition salée, etc.;

- fr. (XVII s.) aller au sel 'acheter des vivres';

- dévéloppements sémantiques du type 'salé' > 'de goût agréable, doux', observables dans plusieurs langues :

- russe sladkij (variante dialectale : solodkij 'doux' et 'bon, goûteux, savoureux') < slave commun *soldŭ-kŭ correspondant à la base i.-eu. *sal-d- 'salé', qui est représentée notamment dans l'angl. salt et l'all. Salz 'sel'; la racine i.-eu. est *sal'sel',

- grec hals 'sel' et 'mer' (> fr. halogène), lat. sal (> fr. sel, salé), russe sol'. Le sens initial en slave : 'salé' et 'ayant du goût, non fade', ensuite 'de goût agréable' et 'doux',

- lituanien saldus 'doux',

- russe solod 'malt' (farine à goût sucré faite d'orge germée artificiellement et séchée, à partir de laquelle on prépare la bière);

- mots i.-eu. signifiant 'doux au goût' et associés étymologiquement à l'idée de « goût piquant» (« qui excite, irrite, pique le palais ») : - lat. dulcis (> fr. doux) et gr. glykys (<*dlukus) 'doux' (> fr. glucose, glycérine, glycine), mots qui seraient apparentés à all. Dolch 'arme blanche, poignard' ;

- racine i.-eu. signifiant 'amer' (*h2em-ro-) qui a donné non seulement le lat. amarus 'amer, âpre, rude, désagréable, pénible, haineux' ( $>$ fr. amer), mais aussi l'albanais ëmbël 'doux' (cf. pourtant en tosque: tëmbël 'fiel, amertume') et 
l'arménien amok 'doux'; développement sémantique analogue à celui observé ailleurs : 'amer' > 'non fade' > 'doux'.

\section{Diachronie « extra-longue » : y aurait-il un rapport entre naître et connaître?}

Même si le rapprochement entre connaître et naître paraît un simple calembour pseudoétymologique (Hugo : « Dans connaitre, il y a naître »), il peut en être autrement lorsqu'on se place dans une très longue perspective diachronique indo-européenne. De ce point de vue, fr. naitre (< lat. nascor < gnascor) serait bien apparenté à connaître (< lat. co-gnosco < (g)nosco) : selon une hypothèse, qui associe i.-eu. * ĝneh $_{3}$ - (Mallory et Adams 1997) 'know, be(come) acquainted with' et i.-eu. *ânh - - 'beget a child ; be born', il s'agirait à l'origine d'une connaissance sociale définie en termes de parenté familiale.

Cf. d'une part lat. nobilis < *gnobilis 'known, glorious ; noble ; excellent ; ill-famed', notus 'known' > nota 'sign, mark', notitia 'fait de connaître qqn; notoriété ; connaissance de qqch., notion, concept ; aveu ; liste ; rapport sexuel', pl. noti 'connaissances, amis' ; et, d'autre part : latin archaïque geno 'engendrer', gigno 'engendrer, procréer', gnascor 'naître' (> nascor), natio 'nation', v. angl. cennan 'engendrer', angl. kind 'genre', all. Kind 'efant', gr. gennâ̂ 'engendrer', genesis 'naissance, origine', genos 'origine; famille, tribuue', gennaios 'propre à telle origine, tel clan ; noble ; excellent'.

Parmi d'autres arguments en faveur de cette hypothèse, on doit tenir compte des liens sémantiques probables, confirmés par des parallèles sémantiques avec différents termes i.-eu. à partir d'autres racines, faits observés à d'autres échelles diachroniques (comme polonais szlachta 'noblesse, aristocratie' < moyen-haut-all. slechte 'espèce, genre, origine', cf. all. Geschlecht 'genre, sexe'). Voici d'autres faits de langues indo-européennes, notamment des parallèles sémantiques importants : russe znat' 'savoir, connaître' et (comme substantif) 'noblesse, gens bien nés' (< i.-eu. *ĝneh ${ }_{3}^{-}$'savoir, connaître'); lat. patricius 'noble, patricien' < pater 'père' ; all. Adel 'noblesse' < i.-eu. *atalo- 'descendance' < *at(i) 'au-dessus' + *al- 'croître ; nourrir' (ou < i.-eu. *at- 'géniteur, aïeul', cf. lat. atavus > fr. atavisme, r. otec 'père') ; serbo-croate plemić 'un noble' < pleme 'tribu'; angl. pedigree < m. fr. pié de grue (pied de grue) 'ramification généalogique' (généalogie notée, connue).

Les parcours sémantiques probables en diachronie seraient les suivants:

- 'naissance, procréation' > 'parenté' > 'fait de (re)connaître qn comme parent, comme membre de son clan' > 'appartenance au clan' > 'noblesse' ;

- 'naissance, procréation' > 'bonne naissance' > 'noble extraction' > 'noblesse, notoriété';

- 'naissance, procréation' > 'parenté' > 'fait de (re)connaître qn comme parent, comme membre de son clan' > 'connaître qqn' ;

- 'appartenance à un clan' > 'bonne naissance' > 'noble extraction' > 'noblesse, notoriété'.

Mais est-ce une raison suffisante pour analyser fr. connaitre comme un dérivé de naître, même en diachronie? Faudrait-il envisager pour les cas de ce type une "protodiachronie »? 


\section{En guise de conclusion}

L'élaboration d'une typologie sémantique qui tiendrait compte à la fois de la variabilité et de la stabilité du rapport forme / sens peut constituer un moyen efficace pour rendre compte des spécificités des langues mais aussi des universaux lexico-sémantiques et de nombreux parallèles sémio-formels. Une double perspective (synchronique et diachronique) y est souvent nécessaire, même si cette possibilité est insuffisamment exploitée jusqu’à présent.

\section{BIBLIOGRAPHIE}

Bottineau Didier, « The submorphemic conjecture in English: Towards a distributed model of the cognitive dynamics of submorphemes ", Lexis $\mathrm{n}^{\circ} 2,2008$, pp. 19-42.

Bottineau Didier, «La submorphologie grammaticale en espagnol et la théorie des cognèmes », in Le Tallec-Lloret Gabrielle (dir.), Vues et contrevues, Actes du XII ${ }^{e}$ Colloque international de Linguistique ibéro-romane, Université de Haute Bretagne - Rennes 2, 24-26 septembre 2008, Limoges, Lambert Lucas, 2010, pp. 19-40.

Bottineau Didier, « Submorphémique et corporéité cognitive », Miranda n 7, 2012, http:// miranda.revues.org/5350.

Franckel Jean-Jacques, Cours de sémantique formelle, Université Paris 7, (texte des notes prises par Sakhno Serguei), 1994.

Hénault-Sakhno Christine et Sakhno Serguei, « Typologie des langues et sémantique diachronique : le problème des universaux ", LINX, $\mathrm{n}^{\circ} 45,2001$, pp. 219-231.

Hénault-Sakhno Christine et Sakhno Serguei, « Typologie sémantique lexicale : problèmes de systématisation », in Lazard Gilbert et Moyse-Faurie Claire (éds), Linguistique typologique. Villeneuve-d'Ascq, Septentrion, 2005, pp. 71-90.

Jalenques Pierre, Contribution à l'étude du préfixe re- en français contemporain : pour une analyse compositionnelle du verbe « regarder », Thèse de doctorat, Université Paris 7, 2000.

Mallory James P. et Adams Douglas Q. (dir.), Encyclopaedia of Indo-European Culture, Chicago, Fitzroy Dearborn, 1997.

Mel'čuk Igor, Cours de morphologie générale, Montréal, Presses de l'Université de Montréal, Paris, CNRS Éditions, 1993-2000.

Nemo François, « Universaux sémantiques : un nouveau champ », Communication au colloque Typologie des langues, universaux linguistiques, 18-19 novembre 2002, Paris, Institut universitaire de France.

Nemo François, « Morphemes and Lexemes versus Morphemes or Lexemes ? ", In on-line Proceedings of the 4th Mediteranean Morphology Meeting (MMM4) on Morphology and Linguistic Typology. (Catania, Sicile, 21-23 Septembre 2003), Booij G, Guevara E, Ralli A, Sgroi S 
and Scalise Sergio (eds), Université de Bologne, 2003. A télécharger sur :http:// morbo.lingue.unibo.it/ mmm/mmm-proc/MMM4/195-208-Nemo-MMM4.pdf

Nemo François, « Routines interprétatives, constructions grammaticales et constructions discursives », Estudos Linguísticos / Linguistic Studies, n 5, 2010, pp. 35-53.

Paulhan Jean, La preuve par l'étymologie, Cognac, Le temps qu'il fait [1953] 1988.

Raccah Pierre-Yves, « Racines lexicales de l'argumentation : la cristallisation des points de vue dans les mots ", Verbum, vol. 32, nº 1, 2011, pp. 119-141.

Rey-Debove Josette, La linguistique du signe, Paris, Armand Colin, 1998.

Robert Stéphane, «Variation des représentations linguistiques : des unités à l'énoncé », in Diversité des langues et représentations cognitives, Paris, Ophrys, 1997, pp. 25-39.

Sakhno Serguei, Dictionnaire russe-français d'étymologie comparée : Correspondances lexicales historiques, Paris, L'Harmattan, 2001.

Sakhno Serguei, « Autour des prépositions russes ob et pro : Problème des parallèles lexicosémantiques slavo - latins ", Slavica Occitania, n 15, 2002, pp. 157-178.

Wierzbicka Anna, «The Salt of the Earth : Explaining the meaning of some of Jesus' sayings in the Sermon on the Mount 2000 », in Iomdin Leonid et Krysin Leonid (eds), Slovo v tekste i v slovare. Moskva, Jazyki russkoj kul'tury, 2000, pp. 61-76.

\section{NOTES}

1. Mais la dérivation diminutive aboutit souvent à des effets difficilement prévisibles : en français, vinaigrette n'est pas «une partie du vinaigre ", car en réalité, ce lexème désigne un mélange d'huile et de vinaigre, salé et poivré, souvent aromatisé. Dans ce cas, le rapport méronymique est pour ainsi dire inversé : en effet, le vinaigre constitue une partie de la sauce qui s'appelle vinaigrette, et c'est de ce point de vue que vinaigrette peut être considéré comme «du petit vinaigre ", c'est à dire du vinaigre non pur, car mélangé d'huile et d'autres additifs.

2. Texte des notes prises par l'auteur.

3. Mais pas de rapport avec fr. soldat (< it. soldato 'celui qui est payé'), fr. solde 'paye', fr. sou, tous remontant au lat. solidus 'massif' et 'pièce d'or massif'. Pas de lien non plus avec fr. salace (< lat. salax 'lubrique').

\section{RÉSUMÉS}

Une typologie sémantique doit tenir compte des régularités de sens, de la variabilité et de la stabilité du rapport complexe forme / sens, ainsi que de l'opacité versus la transparence relative du signifiant. L'analyse dans une double perspective (synchronique et diachronique) y est souvent nécessaire. Cela permet de résoudre certains cas difficiles de reconstructions diachroniques et de décrire des réseaux sémantiques inattendus et considérés souvent comme problématiques. 
A semantic typology must be based on recurring semantic links and it takes in account the lingistic forms and their meanings viewed both in synchrony and diachrony, in a puzzling relation between variability and stability, between opacity and transparency. The typological approach allows to back up some tricky cases of diachronic reconstructions and to describe new semantic networks which may be thought as doubtful.

INDEX

Mots-clés : sémantique, synchronie, diachronie, stéréotypes

Keywords : semantics, synchrony, diachrony

\section{AUTEUR}

\section{SERGUEI SAKHNO}

Université Paris Ouest Nanterre 